\title{
Editorial Commentary to Article "The Magnitude of Blood Pressure Reduction Predicts Poor In-hospital Outcome in Acute Intracerebral Hemorrhage" by Divani et al.
}

\author{
Nils H. Petersen ${ }^{*}$ (1)
}

@ 2020 Springer Science+Business Media, LLC, part of Springer Nature and Neurocritical Care Society

In this issue of the Neurocritical Care, the authors submit an innovative analysis showing that early aggressive reduction in systolic blood pressure is associated with increased mortality and worse in-hospital outcome after intracerebral hemorrhage ( $\mathrm{ICH})$ [1]. The effect may vary depending on initial hematoma volume, and reductions $>40 \mathrm{mmHg}$ may be harmful.

Despite two large randomized trials, the optimal blood pressure (BP) management after ICH remains undefined $[2,3]$. While acute BP lowering is probably of some benefit, there is no evidence that aiming for one particular BP target is broadly advantageous. Furthermore, the majority of subjects in the Antihypertensive Treatment of Acute Cerebral Hemorrhage (ATACH-II) and the Implementation of Intensive Blood Pressure Reduction in Acute Cerebral Hemorrhage Trial (INTERACT-2) had small- to moderate-sized hematomas in subcortical locations, leaving those with large and more severe hemorrhages an understudied population. Thus, characterizing patients that are likely to benefit remains crucial, and the interaction between timing, intensity, and duration of different BP interventions and outcome requires further definition.

To better understand the relationship between systolic blood pressure trajectories and outcome after

\footnotetext{
*Correspondence: nils.petersen@yale.edu

Division of Neurocritical Care and Emergency Neurology, Department of Neurology, Yale Medical School, 15 York St, LCl 1003, New Haven, CT 06510, USA
}

This comment refers to the article available at https://doi.org/10.1007/ s12028-020-01016-Z. intracerebral hemorrhage, the investigators conducted a retrospective study of 757 patients with spontaneous intracerebral hemorrhage (mean age 65 years, median Glasgow coma scale 14). Between 6 and 16 BP measurements were recorded for each patient over the first $24 \mathrm{~h}$ to determine common trends in blood pressure after spontaneous ICH. Using an unsupervised statistical method, the investigators uncovered that mean SBP and SBP reduction within the first $6 \mathrm{~h}$ accounted for $76.8 \%$ of the variation in SBP trajectories.

Although reducing hematoma expansion, reductions in systolic blood pressure were independently associated with higher (worse) scores on the modified Rankin scale (mRS) at discharge (adjusted OR per $10-\mathrm{mmHg}$ 1.13; 95\% CI 1.04-1.23; $p=0.003$ ). Depending on the hematoma size, the shift occurred in different regions of the mRS. For small $(<7.4 \mathrm{ml})$ and medium-size (7.4$30.5 \mathrm{ml}$ ) hematomas, larger reductions in blood pressure were associated with a lower probability of a good outcome (mRS 0-2). In contrast, among patients with large hematomas $(>30.4 \mathrm{ml})$, the shift occurred away from poor (mRS 3-4) toward severe outcomes (mRS 5-6). This differential effect is not surprising given the strong association between hematoma size and outcome with very few patients with large hematomas achieving a good outcome. Furthermore, large SBP reductions $>60 \mathrm{mmHg}$ were associated with a fourfold increase in the risk of death when compared to those $<20 \mathrm{mmHg}(p=0.046)$.

As the authors point out, this study demonstrates an association between BP reductions and outcomes, but it does not prove causality. Information on blood pressure management or the use of antihypertensive medication is

\section{Springer}


not available, and treatment practices likely varied across centers and during the extended timeframe of data collection. Also, the study is limited by missing data and a lack of long-term outcomes. Despite these caveats, this work, along with results of the ATACH-II and INTERACT-2 clinical trials, suggests that a moderate approach to control of hypertension may lead to better outcomes. Furthermore, the findings of this study provide a rationale for considering individual patient factors such as initial hematoma volume and baseline BP levels rather than aiming for one SBP target in all patients. Additionally, this body of work demonstrates the impact of emerging analytical techniques and the potential power of repeated measures of blood pressures to further understand patient-level physiology. While this approach may reveal new insights into the impact of hemodynamics after $\mathrm{ICH}$, the investigators will also need to outline how this understanding can translate into new interventions, whether at the individual or group level.
Conflict of interest

The author reports no conflict of interest.

\section{Publisher's Note}

Springer Nature remains neutral with regard to jurisdictional claims in published maps and institutional affiliations.

Published online: 7 July 2020

References

1. Divani A, Liu X, Lattanzi S, Anderson C, Torbey M, Moullaali T, et al. The magnitude of blood pressure reduction predicts poor in-hospital outcome in acute intracerebral hemorrhage. Neurocrit Care. 2020. https:// doi.org/10.1007/s12028-020-01016-z.

2. Qureshi Al, Palesch YY, Barsan WG, Hanley DF, Hsu CY, Martin RL, et al. Intensive blood-pressure lowering in patients with acute cerebral hemorrhage. New Engl J Med. 2016;375:1033-43.

3. Anderson CS, Heeley E, Huang Y, Wang J, Stapf C, Delcourt C, et al. Rapid blood-pressure lowering in patients with acute intracerebral hemorrhage. New Engl J Med. 2013;368:2355-65. 